\title{
Aerodynamic Sensing as Feedback for Ornithopter Flight Control
}

\author{
Derrick Yeo ${ }^{1}$, Ella M. Atkins. ${ }^{2}$, and Wei Shyy. ${ }^{3}$ \\ University of Michigan, Ann Arbor, Michigan, 48109, U.S.A
}

Flapping wing vehicles, or ornithopters, have proven difficult to control due to the unsteady flow generated by the high-speed flapping surfaces. To-date, research has focused on computational models from which fixed flapping strokes are optimized. These strokes are then fixed and executed open-loop in practice with flapping speed as the primary control output for climb and descent. This paper investigates the use of a distributed pressure sensing system embedded in the flapping wing surfaces to provide real-time aerodynamic force estimates. These measurements could ultimately be used as a source of feedback for an ornithopter autopilot system. This paper describes the design, construction, and testing of flat plate and airfoil ornithopter wings into which pressure lines were embedded during construction. The embedded pressure lines were tethered to external highprecision pressure sensors, while the wings were mounted to a commercially-available ornithopter body then affixed to an instrumented flap stand. A series of exploratory low-speed wind tunnel tests were conducted during which pressures, airspeed, wing deflections, and overall forces/torques were acquired. Initial data is consistent and is observed to match trends obtained from a panel method simulation used to generate comparative pressure measurements over the flapping stroke.

\section{Nomenclature}

$\begin{array}{ll}\mathrm{b} & =\text { wingspan, } \mathrm{m} \\ \mathrm{c} & =\text { chord, } \mathrm{m} \\ h & =\text { stroke amplitude, } \mathrm{m} \\ f & =\text { frequency of oscillation, } \mathrm{Hz} \\ U_{\infty} & =\text { free stream velocity, } \mathrm{m} / \mathrm{s} \\ \operatorname{Re} & =\text { Reynolds number, } \mathrm{Re}=\frac{V_{t i p} \cdot c}{v} \\ S t & =\text { Strouhal number, } S t=\frac{h \cdot f}{U_{\infty}} \\ \mathrm{V} & =\text { velocity, } \mathrm{m} / \mathrm{s} \\ \beta & =\text { wing flapping angle } \\ v & =\text { kinematic viscosity, } \mathrm{m}^{2} / \mathrm{s} \\ \phi & =\text { velocity potential }\end{array}$

\section{Introduction}

URRENT flapping wing vehicles rely on moving surfaces adapted from traditional aircraft designs for attitude control. A single tail surface typically provides pitch and directional control and the main wings are not actuated beyond their predetermined flapping stroke, albeit at a controllable flapping frequency. ${ }^{1}$ While flapping flight has been successfully demonstrated, existing ornithopter designs do not offer the agility of fixed or rotary wing vehicles. Furthermore, it has been found that motion of the single tail surface found on most ornithopters varies moments about multiple axes, resulting in an inherent flight control coupling that makes attitude

\footnotetext{
${ }^{1} \mathrm{PhD}$ student, Aerospace Engineering Dept, University of Michigan, Ann Arbor, MI

${ }^{2}$ Associate Professor, Aerospace Engineering Dept, University of Michigan, Ann Arbor, MI, Associate Fellow.

${ }^{3}$ Clarence L. "Kelly" Johnson Collegiate Professor, Department of Aerospace Engineering, University of Michigan; currently, Provost \& Chair Professor, Department of Mechanical Engineering, Hong Kong University of Science and Technology
} 
control a challenging task despite the low potential for maneuverability. ${ }^{2}$ Incorporating attitude control actuation on the moving wings of an ornithopter presents physical and modeling challenges due to constantly changing forces and inherently non-linear aerodynamics. However, if such actuation were available, highly-maneuverable or even aerobatic ornithopter flight might be realized. Rotorcraft have successfully managed constantly moving blades for over 75 years but their mechanisms are not easily transferred to ornithopter flight. Ornithopter aerodynamic surfaces are large relative to their actuation speed, so the relationships between mechanical actuation and resultant aerodynamic forces are highly dependent on external flow conditions. Given suitable flow sensing and aerodynamic

modeling capabilities, real-time ornithopter feedback control could be realized with a more articulated, tunable wing stroke than has been previously utilized.

In this paper, we present efforts to sense flow over flapping wings in a manner that ultimately could support realtime feedback control in an un-engineered indoor or outdoor flight environment. To do this, we designed, constructed, and instrumented flat plate and airfoil wings with a series of pressure ports capable of providing realtime estimates of the aerodynamic forces generated by the flapping wing surfaces. We adapted a pressure sensor suite developed previously ${ }^{3}$ to construct a first-generation tethered sensing system in which miniature pressure lines embedded in the flapping wings are routed off-board to high-precision pressure sensors and high-speed data acquisition electronics. We also measured airspeed, real-time deflection of the wings, and total force/torque. Data acquired during a series of slow-speed wind tunnel tests in which the flapper was affixed to a wind tunnel mount with force/torque sensing capability are analyzed to identify the relationship between pressure measurements, inertial measurements (attitude and rates), and induced forces and moments as well as sensitivity to different wind conditions and potential actuation profiles.

While our final goal is to create a flapping wing flight controller, this study first explores the possibility of using aerodynamic feedback on flapping wing craft. Below, we first present a wing actuation concept that motivates the use of real-time aerodynamic force feedback to an autopilot that could take advantage of multiple flapping wing actuation degrees of freedom. Next, we describe the design, construction, and integration of highly-instrumented elliptical flat plate and airfoil flapping wings used throughout our tests. We then review a simple aerodynamic model used to motivate our measurements and validate observed trends in our datasets. After a description of the flap stand and wind tunnel setup, a series of results are presented from our initial series of flapping wing tests in the University of Michigan's low-speed 5'x7' wind tunnel. Tests included zero (hover) and low free stream velocities that might be expected for a small flapping vehicle of the scale we tested.

\section{Motivating Actuation Concept}

Flapping wing flight offers new possibilities in terms of actuation over conventional control surfaces such as elevons. With flapping, the main wings create both lift and thrust. Altering aerodynamic properties such as camber on each wing can vary the amount of thrust produced on either side of the vehicle $\mathrm{e}^{4,5}$ analogous to actuation of the B2 bomber drag rudder. Since the wings are hinged, it is also possible for an ornithopter to vary its flap angle on either wing, rotating the resulting lift vector of both wings differentially between the two sides. Further, a flapping wing vehicle inherits the ability to vary its dihedral angle in a glide by virtue of its hinged wings as well as its mean flap angle between both wings when in a flapping configuration. By independently varying the mean flap angle of each wing, a control strategy that is analogous to tilting the tip path plane of a helicopter is possible. This is depicted in the schematic below. 


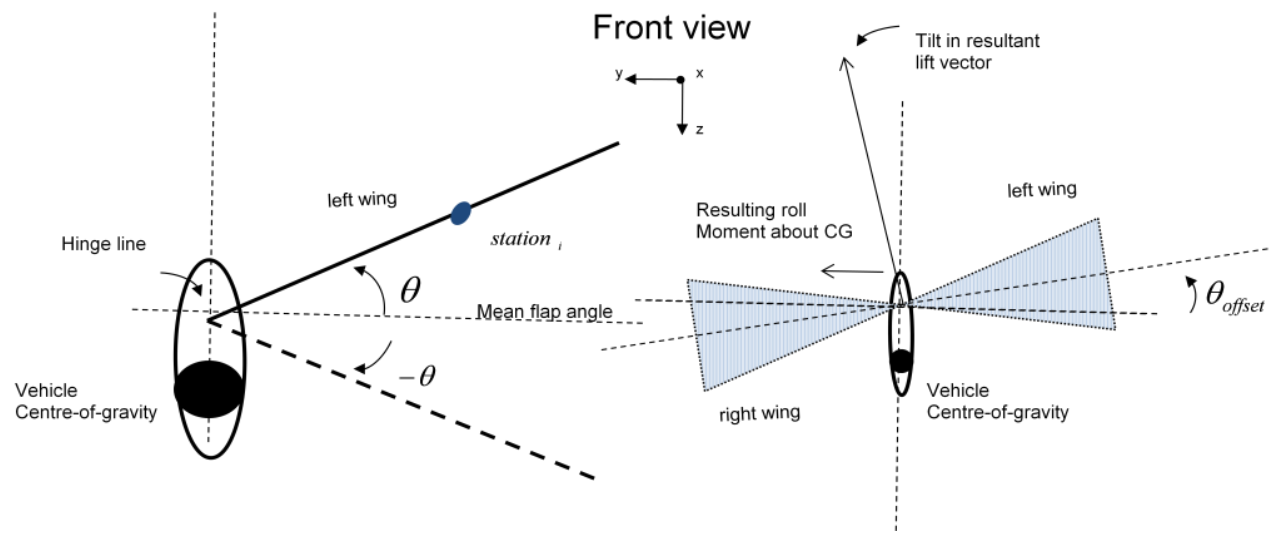

Figure 1: Potential Method for Controlling Roll Through Mean Flap Angle.

These concepts have been studied by Paranjape et al in relation to a tailless bio-inspired micro air vehicle with articulated wings. ${ }^{6}$ Vehicle controllers have been developed that take advantage of dihedral control. ${ }^{7}$ Another strategy is to actively control the shape of the wings in flight using smart materials such as Piezoelectric actuators. ${ }^{8}$ Shape control allows smooth variation of wing camber given flexible wings. A schematic of one such implementation is shown below from Kim et al. ${ }^{9}$

Once more complex actuation strategies are implemented, accurate control of all actuation degrees of freedom, especially in variable flow conditions (e.g., gusts), will be made possible through real-time feedback. Although rigid body inertial sensing is beneficial, the flapping wing platform is far from a rigid body, suggesting that direct measurements of forces or torques may also be beneficial. With the assumption that flow visualization would not be a viable means of estimating forces for a deployed ornithopter, we hypothesize that sensing of pressures distributed across the flapping surfaces can also provide a good approximation of the overall forces generated during flapping. Since existing commercially-available pressure sensing and high-speed data acquisition equipment cannot be flown on a small ornithopter, we instead tether the ornithopter to an external sensing system. The authors have previously deployed a pressure-based aerodynamic sensing system in a larger fixed-wing platform and validated its performance in flight testing. ${ }^{3}$ Although the platform and measurements substantially differed in this work, the previous experiences demonstrated that real-time distributed pressure measurement in flight was indeed possible provided the supporting avionics can be miniaturized further.

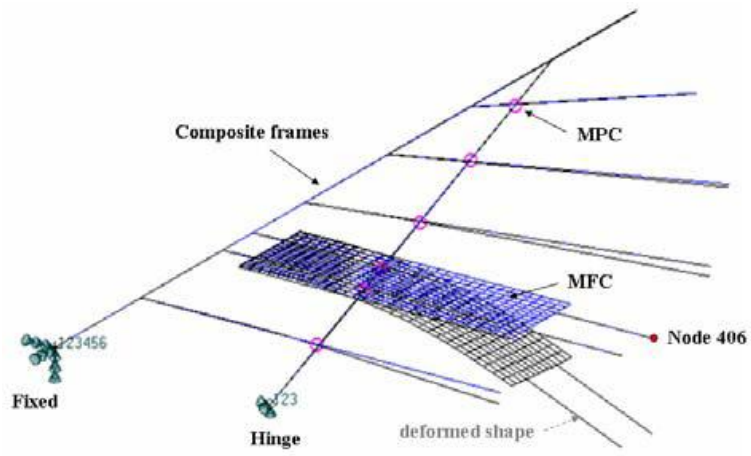

Figure 2: Schematic of a MFC Actuator On a Flexible Wing (Kim et al.) ${ }^{9}$

\section{Instrumented Flapping Wings}

The purpose of the described actuation scheme is to effect changes in resultant aerodynamic forces in a manner that offers more maneuverability than that offered by conventional methods. However, the relationships between actuation time histories and these forces are largely dependent on ambient flow conditions. For a successful control strategy to be implemented, a suitable sensor solution is needed. In previous work, Grauer and Hubbard have described a mainly inertial sensor suite tailored to flapping wing vehicles. ${ }^{10}$ A two meter span flapping wing platform has been instrumented with inertial sensors and flown by Jackowski and Tedrake. ${ }^{11}$ To enable characterization of locally-generated aerodynamic forces, we propose a flow sensor package that will enable an 
autopilot to take advantage of an advanced actuation scheme as well as compensating for observed flow conditions and disturbances. Sensing pressures, our strategy in this work, will enable estimation of the total aerodynamic forces being generated as well as measuring differences in loading between the left and right wing. In previous work, we investigated the use of embedded pressure sensors on a small hovering fixed-wing aircraft to provide real-time estimates of vehicle aerodynamic state. ${ }^{3}$ Results from this effort were promising, although the avionics to support this test setup were too large in volume and weight to be hosted on a free-flying small flapping vehicle. We therefore migrated the sensors, power systems, and data acquisition electronics offboard for our flapping wing tests in which the flapping vehicle was affixed to a flap stand and tethered through pressure and power lines, as will be described below. Other groups are also investigating the use of differential pressure sensing to characterize flapping flight, with efforts leading to differential pressure measurement systems that have actually been flown ${ }^{12}$. That these sensors are within the current state of the art suggests that our pursuit is not purely academic. Our focus is complementary. With our sensor suite, we seek to obtain the highest-quality distributed pressure data possible with external sensors and data acquisition, correlate this data with aerodynamic model predictions and implement a working controller within a wind tunnel environment. We will then migrate to lightweight sensors that ultimately could be carried onboard an ornithopter in free flight.

\section{Flapping Wing Design}

To accommodate the pressure ports and lines used for our tests, the flapping wings were custom designed and fabricated. We constructed two pairs of custom wings as shown in Figures 3 and 4: one set of rectangular planform wings with a NACA0015 airfoil section, and a set of elliptical, planar wings more typically found in flapping wing vehicles. These wings, constructed of balsa wood, were built to be rigid at the expected flapping frequencies. Use of a rigid wing structure enabled us to focus our efforts on simplified aerodynamics rather than flexing behaviors. The wings were attached to an off-the-shelf Cybird flapping mechanism using custom adapter cylinders used to manually adjust incidence angle of the wing root. To prevent wing rotation about the feathering axis, rearward mounting hinge plates were crafted from thick vinyl.
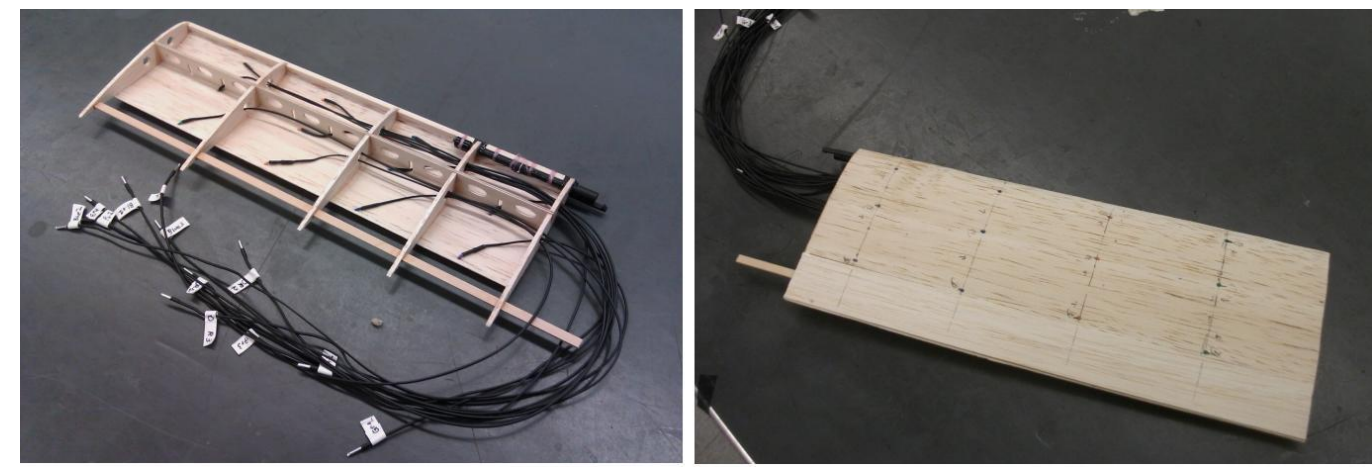

Figure 3: Instrumented NACA 0015 Flapping Wings.
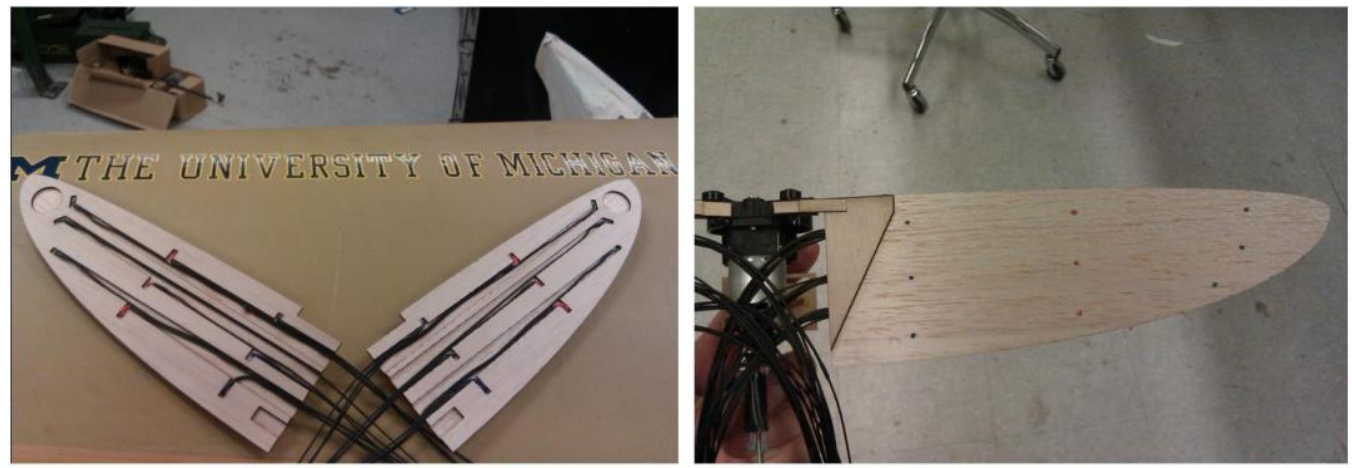

Figure 4: Instrumented Airfoil Flapping Wing Pair. 
Pressure ports were installed in both wing pairs as shown in the above figures. This regular spacing was also modeled in our numerical aerodynamic load estimation strategy, described below. Each flapping wing was discretized into areas with the aerodynamic pressure on each area represented by measurements taken at their center. As such, pressure ports were located at three span-wise positions on the elliptical wings. The larger NACA 0015 wings were equipped with pressure ports at four span-wise locations. Each location on both wings had three pressure ports. These locations and their areas of presumed coverage are shown in the schematics illustrated below.

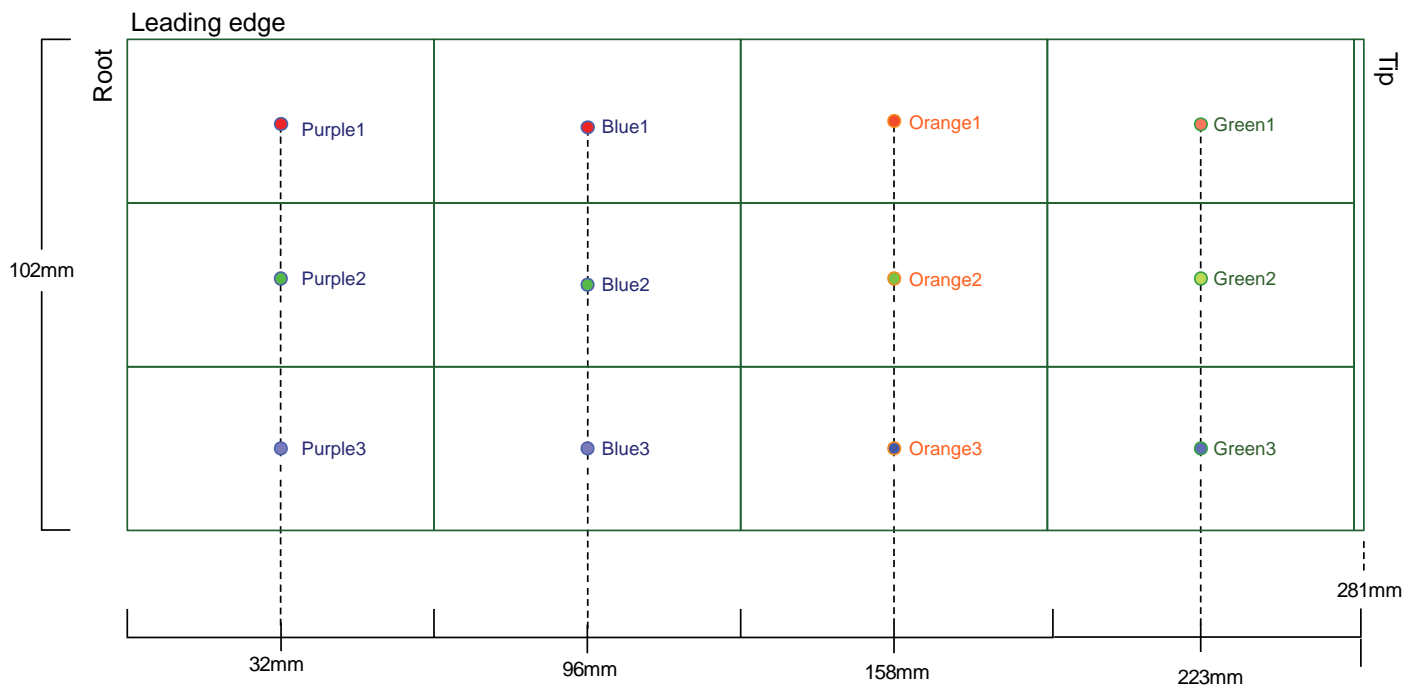

Figure 5: NACA0015 Wing Dimensions and Pressure Port Locations.

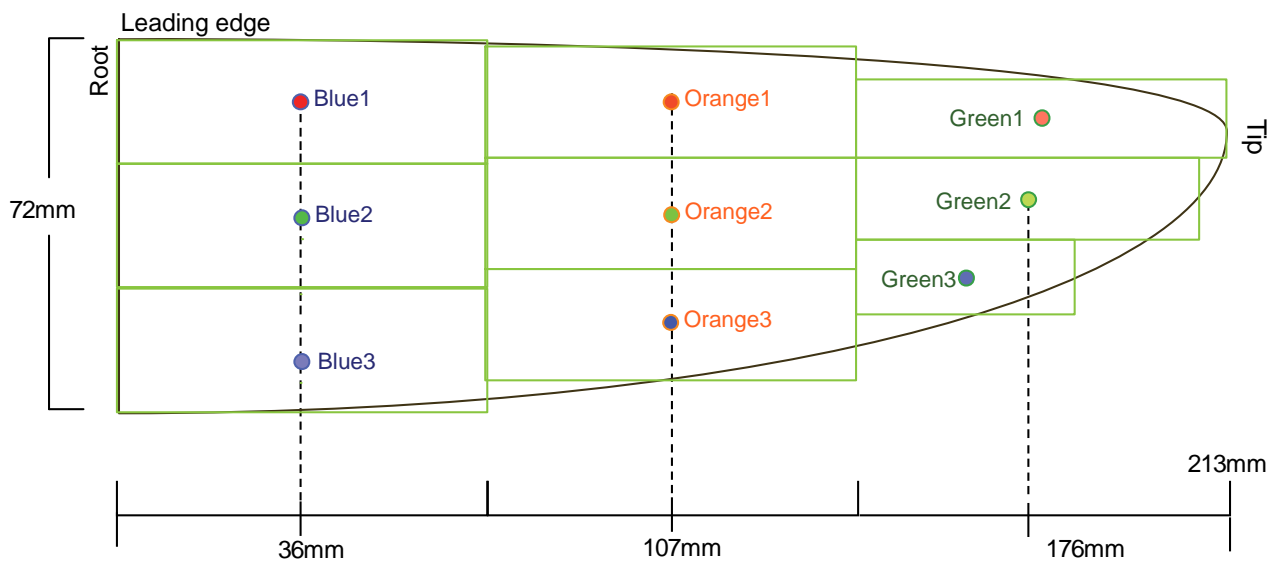

Figure 6: Flat Plate Wing Dimensions and Pressure Port Locations.

The pressure ports were colour coded and organized by their relative positions on the wing chord as shown above. Each port location contains two ports, one on top and one directly below it. Differential pressure readings are thus taken across the top and bottom surface at these locations. The ports locations are numbered from one to three based with port1's closest to the leading edge. In the data plots that follow, port1 data is plotted in red, port2 data is green and port 3 data is given in green.

\section{Numerical Predictions of Flapping Wing Pressure Trends}

To assess the validity of our pressure measurement scheme, a simple aerodynamic model was developed to predict qualitative trends in data. A quasi-3D vortex method solver was written in Matlab to provide a simplified overview of pressure trends over a flap stroke; this model served as a first order check of test results. In a vortex method formulation, the wing surface is represented by a distribution of bound vortices and the resulting flow field is 
determined using potential flow methods. The solver used is based on the 2D method outlined in a text by Katz ${ }^{12}$ and represents a limited but simple solution to generating a 'preview' of the anticipated test data. The following section provides a brief overview of the basic concepts behind potential flow methods. Discrete unsteady vortex method codes such as those developed by Ansari ${ }^{14}$, Katz ${ }^{15}$ and Willis ${ }^{16,17}$ are highly sophisticated and elegant solvers that are capable of tracking both shed vorticity from the trailing edge and also the leading edge in cases of fully separated flow. Additional details can be found in the aforementioned references.

\section{Governing Equations and Boundary Conditions}

A vortex element method is an example of a potential flow solver that assumes incompressible and inviscid flow. The continuity equation for incompressible flow is given by

$$
\nabla \cdot \bar{V}=0
$$

where $\bar{V}$ represents the flow velocity vector. Defining the velocity potential $\phi$ as $\bar{V}=\nabla \cdot \phi$, this results in

$$
\nabla \cdot \nabla \cdot \phi=\nabla^{2} \phi=0
$$

As such, continuity is satisfied using Laplace's equation. As a linear differential equation, elementary solutions of Laplace's equation can be combined to form solutions subject to boundary conditions. If $\Phi_{1}, \Phi_{2} \ldots \Phi_{n}$ are solutions of the Laplace Equation (1) then

$$
\Phi=\sum_{k=1}^{n} c_{k} \Phi_{k}
$$

is also a solution of the Laplace equation. Since the $c_{k}$ represent arbitrary constants, Equation (2) can be written as

$$
\nabla^{2} \Phi=\sum_{k=1}^{n} c_{k} \nabla^{2} \Phi_{k}=0
$$

Common elementary solutions include sources, sinks, doublets, and vortices. With respect to flapping flight, vortex distributions are used to represent a lifting surface or wing section and where applicable, the shed wakes from both the leading and trailing edges. A number of boundary conditions apply. For example, flow can only be tangential to surfaces, the Kutta condition. Geometrical constraints are enforced to provide a system of linear equations. In the most basic formulation, these equations are solved to provide the vortex distribution of a given set of flow conditions. While vortex methods are not limited to $2 \mathrm{D}$, one common approach is to perform a blade element discretization of the wing span by treating each chord section as one 2D problem. In this quasi-2D approximation, each section is subject to the local flow conditions that arise from wing kinematics, the incident free stream, and the wake or wakes that associated with that particular wing section.

\section{Qualitative Vortex Method estimates}

The discrete vortex solver that was written and used for this work calculates quasi-steady loads but does not track shed vorticity and assumes fully-attached flow. This approximation is sufficient for its intended purpose of providing qualitative results that are easily accessible while remaining relevant for trend prediction. Once our calculations were made, we extracted pressure values at certain points across the wing to represent our distributed pressure sensor readings. These were used to generate pressure histories across a sinusoidal flap cycle with wings at zero incidence angle to provide expected trends. The case shown below was dimensioned to give a Strouhal number of approximately 0.15 . This was chosen to provide trends that would be consistent with the thick rounded NACA 0015 flapping airfoil which would see largely attached flows at lower Strouhal numbers. 

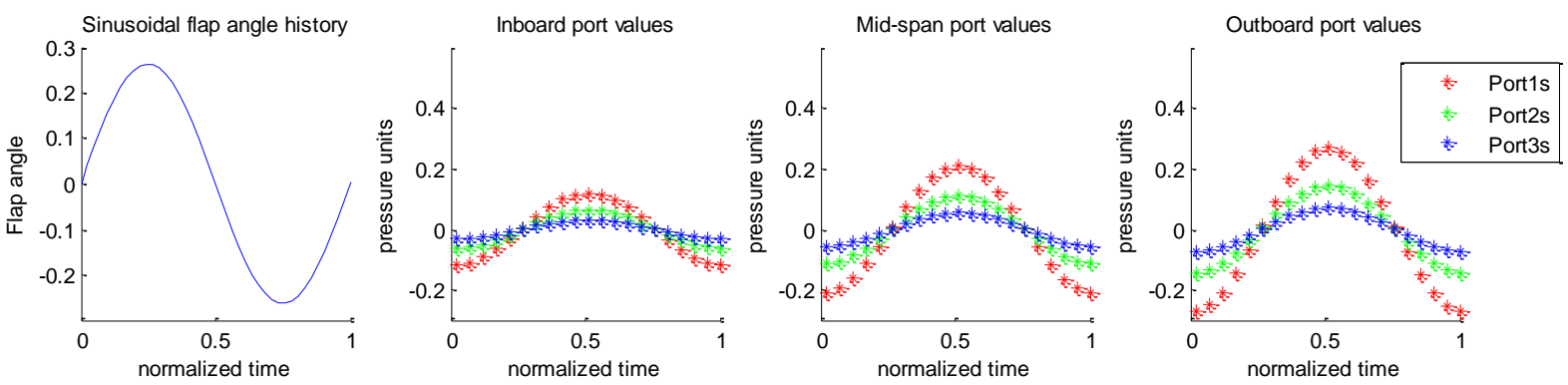

Figure 7: Flap Cycle Pressures Predicted from a Discrete Vortex Solver at Zero Incidence.

The red, green, and blue trends indicate values that represent readings from the leading, middle and trailing pressure ports respectively at each of the wing sections. The theoretical values showed trends that were expected of a symmetrical flap stroke where positive pressure peaks were equal to the negative pressure peaks. The larger instantaneous velocities at outboard stations also resulted in scaled up values towards the tip. The attached flow formulation predicted large suction peaks near the front of the airfoil.
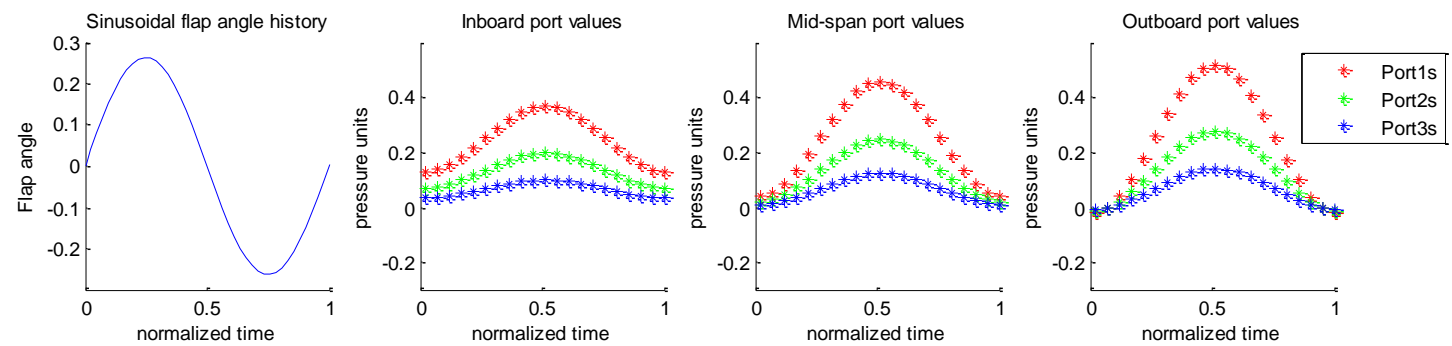

Figure 8: Qualitative Results from a Simple Discrete Vortex Solver, Positive incidence.

A similar case was run with a small positive angle of incidence, showing variations in higher positive pressure peaks on the downstroke and diminished pressure values on the upstroke as expected. Close to the root, it can be noted that the pressure distribution is always positive and oriented upwards. From this data it appears possible for the leading stations to always generate a positive lifting condition. While approximate, these results provide a simple check by which the experimental pressure measurements can be validated. For a higher-fidelity quantitative comparison, the use of an unsteady code that handles both leading and trailing edge separation is planned as part of our future work.

\section{Wind Tunnel Test Setup}

Wind tunnel testing was performed to enable evaluation of the flapping wing measurements over multiple free stream flow conditions. As described above, the Cybird ornithopter mechanisms used for this testing constrained the wings to a fixed flap stroke at variable speeds, but our attachment mechanisms enabled us to manually adjust left/right differential pitch. More specifically, pitch control was achieved through custom wing root mounts that adapted to the Cybird wing mounts but e mounted to the wings at different angles using spacers. In addition to the pressure-based aerodynamic data system, a six axis force-torque sensor and a pair of infrared range sensors were also used for data acquisition, as shown in Figure 10. In this figure, the infrared sensor pair used to measure wingtip distance from the base plate is shown on the left, while the flap stand and test ornithopter with flat plate wings affixed are shown in multiple views. All real-time data was analog, acquired through a PC/104 data acquisition system at up to $3 \mathrm{kHz}$ using a Diamond Systems Athena embedded computer and auxiliary DMM-32 data acquisition board also attached to the $\mathrm{PC} / 104$ bus.

An ATI Nano-17 force-torque sensor was utilized, with recorded strain gauge values converted during data acquisition to force-torque readings using calibration software provided by ATI. Infrared sensors were used to provide a measurement of each wing's motion to provide a measure of flapping wing motion independent of pressure data. This addressed issues previously encountered when we attempted use of a a rotary encoder on the ornithopter mechanism. The encoder was unable to account for mechanical play in the transmission, the effects of 
which varied between low and high frequency runs. At higher flapping rates, the free play in the transmission allowed the wings to lag behind the transmission and alter the shape of the flapping angle curve. These sensors presented challenges of their own due to the relatively long measurement distances and relatively short range of recorded values involved, but they provided sufficiently clean data for analysis as will be described below.

The flap stand was configured to fit inside the wind tunnel test section, reducing the need for long pressure line and analog signal wire runs. This was an advantage that our embedded computer and sensor system offered. As shown in the above photos, the pressure sensor bank, computer and FT sensor amplifier were positioned downstream of the main stand. High performance polycarbonate radio controlled race car body shells were used as aerodynamic fairings to minimize flow disturbance as well as exposure of electronics to wind tunnel flow. These were low cost, readily available and supplied in a transparent, unpainted form which allowed visual inspection of the pressure fittings, wiring, and computer stack. The pressure sensors were re-calibrated prior to wind tunnel testing using inclined manometers and checked against the generic manufacturer calibrations. The FT sensor was pre-calibrated by ATI and the supplied calibration data was loaded on the Athena computer; correct FT sensor operation was also verified using calibrated weights. The pressure ports were verified to be clear of obstructions and a strain relief line guide prevented flapping motions from cinching or deforming the pressure lines during testing. Relative component placement is shown in the schematic and pictures below.

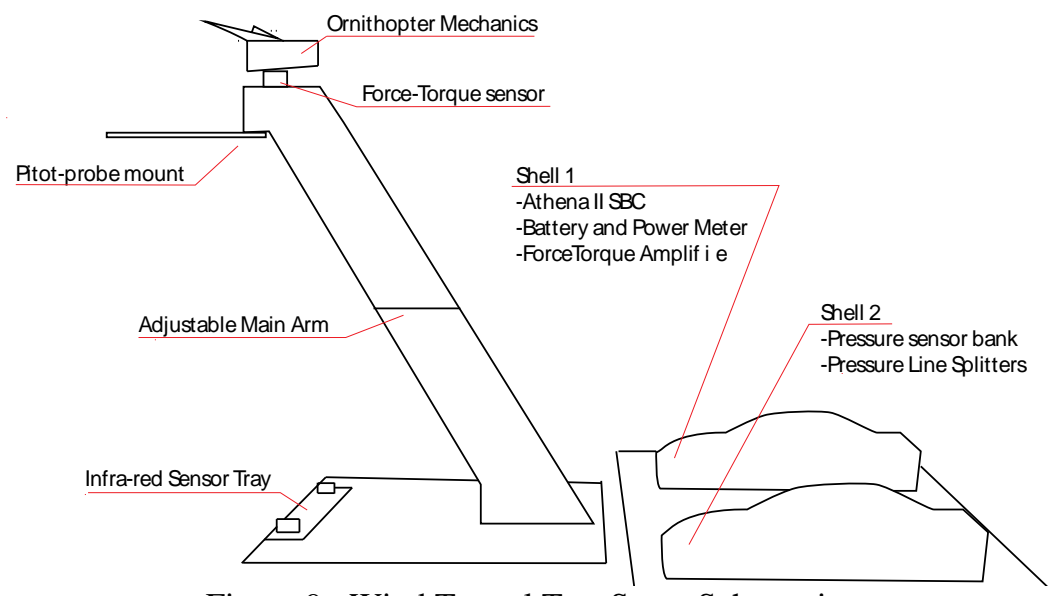

Figure 9: Wind Tunnel Test Setup Schematic
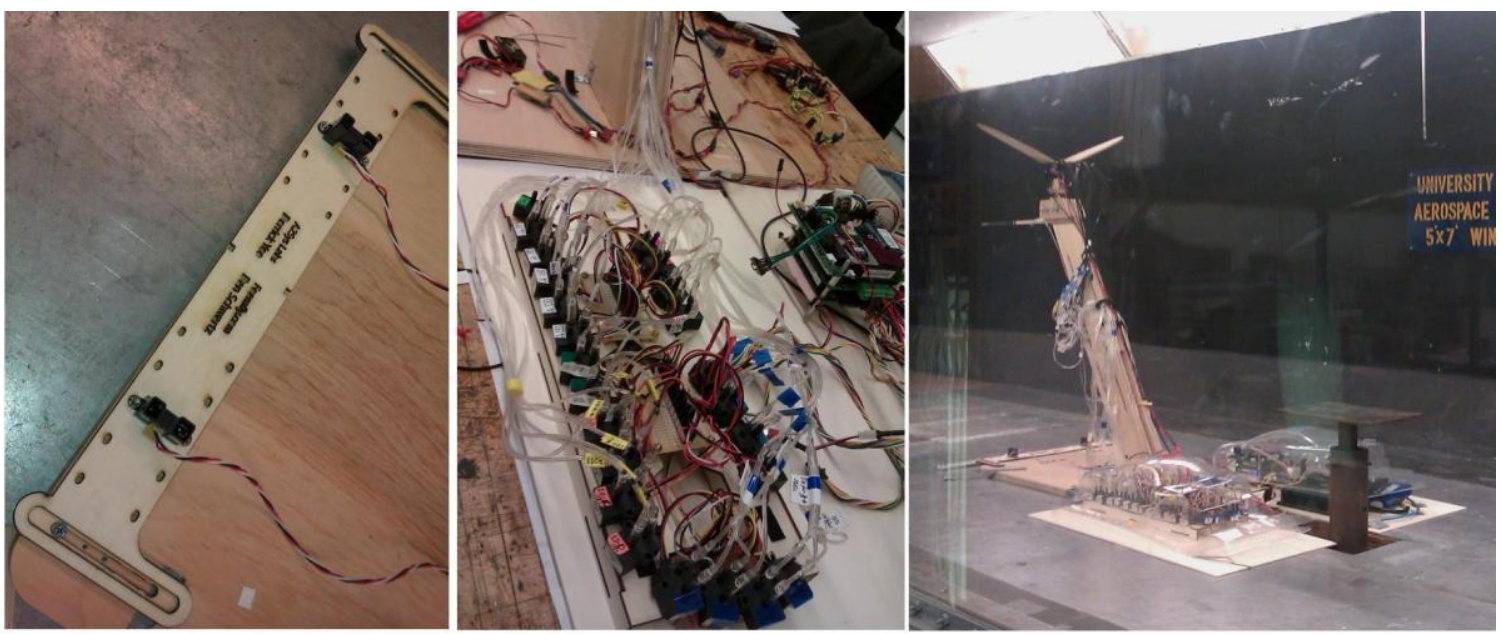

Figure 10: Infra-red Sensor Tray, Pressure Sensor Bank, and Overall View

\section{Test Results}


A number of exploratory tests were performed over different Strouhal numbers and flapping wing pitch angles. Time in a vacuum chamber was secured to isolate inertial loads but due to logistical reasons, these tests could not be carried out in time for this manuscript. The free stream of the wind tunnel was fixed for all the tests and the Strouhal number was changed by varying the flap frequency. An upper bound on wing/mechanism operating frequency was determined by destruction testing a set of quickly built uninstrumented wings.

A free stream velocity of approximately $3 \mathrm{~m} / \mathrm{s}$ was chosen to afford a spread of Strouhal numbers within what we considered to be our effective frequency range. At free streams in the range of $3 \mathrm{~m} / \mathrm{s}$, the turbulent intensity of the wind tunnel was calculated to be between $2-4 \%$. The following table describes our test matrix for conditions with a non-zero free stream and provides a map of the subset of cases presented in this paper. Test names in bold indicate cases that have been considered of most interest and are presented for comparison.

Table 1: Naca0015 Rectangular Wing Test Series

\begin{tabular}{|l|c|c|c|}
\cline { 2 - 4 } \multicolumn{1}{c|}{ Naca0015 } & \multicolumn{3}{c|}{ Approximate St number: } \\
\cline { 2 - 4 } \multicolumn{1}{c|}{} & $\mathrm{St}=0$ & $\mathrm{St}=0.12$ & $\mathrm{St}=0.15$ \\
\hline Wing Alpha $=5 \mathrm{deg}$ & testN1 & testN2 & testN3 \\
\hline Wing Alpha $=10 \mathrm{deg}$ & testN4 & testN5 & testN6 \\
\hline
\end{tabular}

Table 2: Flat Plate Elliptical Wing Test Series, No Free-stream

\begin{tabular}{l|l|l|l|l|}
\hline \multirow{2}{*}{$\begin{array}{l}\text { Elliptical Wing - } \\
\text { Hover }\end{array}$} & \multicolumn{3}{|l|}{ Flap Frequency } \\
\cline { 2 - 5 } & $1 \mathrm{hz}$ & $2 \mathrm{hz}$ & $3 \mathrm{hz}$ & $3.5 \mathrm{hz}$ \\
\hline testH1 & testH2 & testH3 & testH3b \\
\hline
\end{tabular}

Table 3: Flat Plate Elliptical Wing Test Series, 3m/s Free-stream

\begin{tabular}{|c|c|c|c|c|c|}
\hline \multirow[t]{2}{*}{ Elliptical Wing } & \multicolumn{5}{|c|}{ Approximate St number: } \\
\hline & $\mathrm{St}=0$ & $\mathrm{St}=0.1$ & $\mathrm{St}=0.2$ & st $=0.25$ & St $=0.3$ \\
\hline Vehicle Alpha = 0deg & testA & testB & testC & testD & testE \\
\hline Vehicle Alpha = 4deg & testF & testG & testH & testl & testJ \\
\hline Vehicle Alpha = 8deg & testK & testL & testM & testN & testO \\
\hline
\end{tabular}

Initial testing with differential pitch actuation was also performed, and while initial results appear to be encouraging, useful data processing and analysis will not be possible until the inertial loads can be measured in vacuum chamber testing thus subtracted from overall forces and torques.

Table 4: Flat Plate Elliptical Wing, 3m/s Free-stream with differential incidence

$$
\begin{aligned}
& \text { Elliptical wing configuration: } \\
& \text { Stroke plane inclination }=4 \mathrm{deg} \\
& \text { Left wing incidence }=+4 \mathrm{deg} \\
& \text { Right wing incidence }=-4 \mathrm{deg}
\end{aligned}
$$

\begin{tabular}{|l|l|l|}
\hline \multicolumn{2}{|c|}{ Approximate St number } \\
\hline $\mathrm{St}=0$ & $\mathrm{St}=0.1$ & $\mathrm{St}=0.2$ \\
\hline TestDP1 & TestDP2 & TestDP3 \\
\hline
\end{tabular}

Acquired data was compiled and ensemble averaged across multiple flaps to generate typical stroke histories for wing displacement, pressure, and force measurements at different conditions. The point where the wing passes through horizontal on the upstroke was chosen to be the indication of a new flap cycle. Infrared (IR) sensor data presented a challenge due to its relatively noisy signal. A successful data processing technique was found that involved first applying a median filter to the IR data to reduce spikes in data. A polynomial curve fit was then applied and conditioned at stroke end points. Since the wings were rigid, these measurements were easily converted 
to wing flapping angle at the root and then compared against the trends given by a four-bar simulator written in Matlab for the Cybird mechanism. Once this was done, a final fourth-order Fourier series fit was applied to the conditioned polynomial fit to provide a periodic function for flap angle in the form

$$
\beta(t)=a_{0}+a_{1} \cos (t w)+b_{1} \sin (t x)+\ldots .+a_{n} \cos (n \cdot t w)+b_{n} \sin (n \cdot t w)
$$

This was checked against the measured motion ranges of the mechanics for soundness of fit. The figure below plots sample measured sensor displacements overlayed with calculated displacements from its corresponding Fourier series fit.

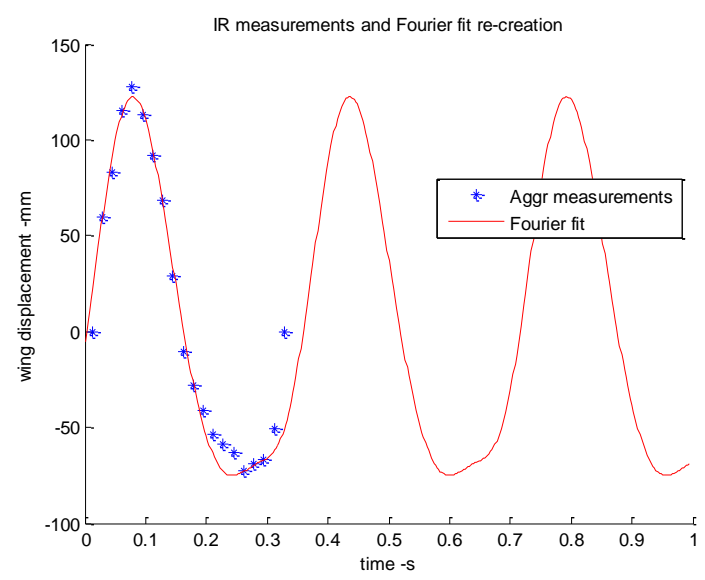

Figure 11: Wing Stroke from Sample IR measurements and a Fourier Series Fit.

Since the actual deflections were measured, this process resulted in a flap stroke that accounted for mechanical play in the transmission and its effects at different frequencies. However, the current set of noisy IR sensors were considered to be a weak link in the experiment and are thus candidates for upgrading in future testing. Future improvements to the test setup will include a bank of more than two IR sensors in addition to more reliable flap mechanics.

\section{NACA 0015 Airfoil Pressure Data}

The rectangular wings with a symmetrical NACA0015 airfoil section were built to represent a more straightforward, predominantly attached flow test case that would allow a first order verification of the test setups performance. The follow composite figures show data from a NACA0015 test wing undergoing a $1 \mathrm{~Hz}$ flap motion in a 3 m/s free stream. The flapping angle history is presented for each test run followed by pressure reading histories. Pressure measurements are differential between top and bottom, where a positive reading indicates higher pressure on the underside of the wing. Readings from the same chord section are presented on the same plot with values from the different chordwise positions plotted in different colors. The data on the left is for a NACA0015 wing at 5 degree incidence while the right hand column shows the data for the airfoil section at a higher 10 degree angle of incidence. 

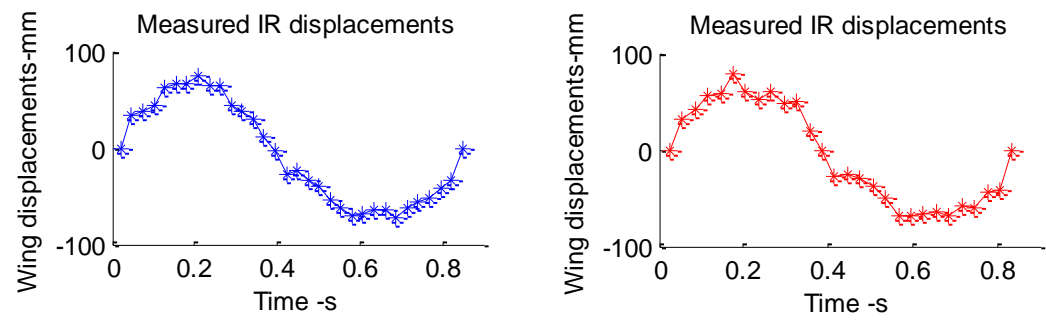

5 deg Alpha blue ports

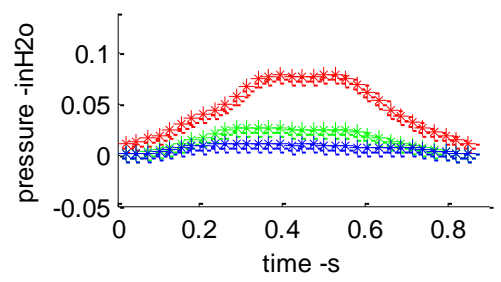

10deg Alpha blue ports

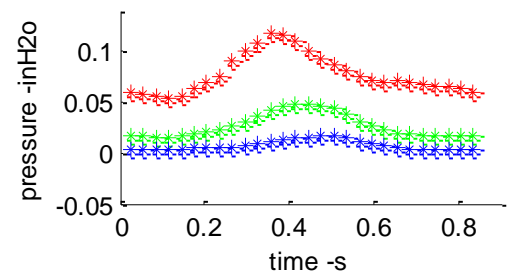

5deg Alpha Orange ports

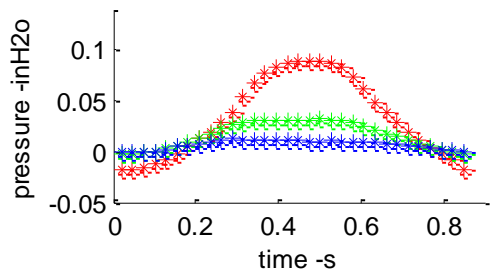

10deg Alpha Orange ports

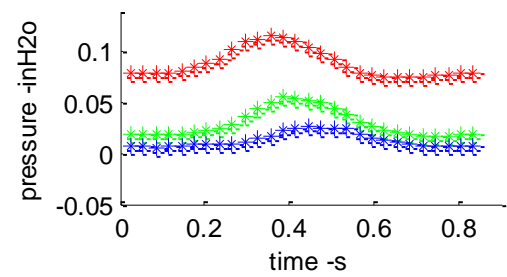

5 deg Alpha green ports

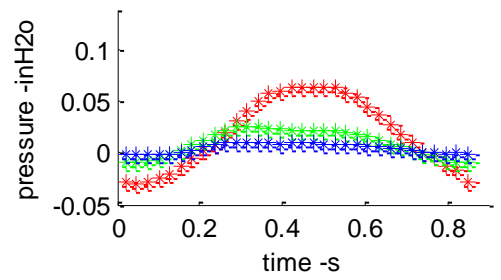

10deg Alpha green ports

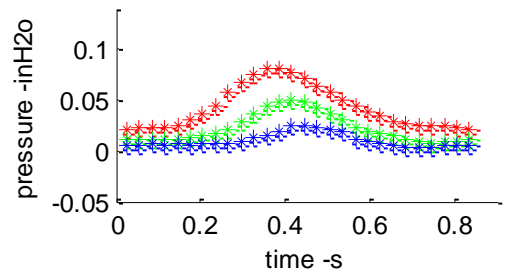

Figure 12: NACA 0015 Pressure Time Histories.

The increase in pressure magnitudes with increasing span at $1 \mathrm{~Hz}$ can be noted from the blue to orange sections. This is expected due to the larger instantaneous velocities encountered further outboard. These observations are intuitive and match the trends predicted by the simple vortex solver. It is also observed that at the blue sections, the lower instantaneous velocities encountered are not sufficient to cause the section to create negative lift given the baseline $5 \mathrm{deg}$ angle of incidence. This observation also matched the trends shown by the simple solver. At the higher $10 \mathrm{deg}$ angle of incidence, the wing was noted to be creating lift throughout the stroke. The readings observed in stationary wing tests show slightly lower suction peak values at the outboard sections suggesting the effect of tip losses. Also when flapping at $1 \mathrm{~Hz}$, the peak magnitudes on the outboard ports are slightly smaller than those measured at the middle chord section, contrary to the predictions made by the quasi $2 \mathrm{D}$ numerical solution. This is most likely due to the onset of flow separation and 3D tip losses at larger local flow angles and loading.

\section{Elliptical wings}

With confidence in the pressure system, the flat plate airfoil elliptical wings were next mounted. An elliptical planform with a planar cross section was chosen as a better representation of a practical flapping wing. These wings were tested both at conditions with no free stream (hover) and at a free stream of approximately $3 \mathrm{~m} / \mathrm{s}$. The resultant forces and torques generated by the vehicle were also measured in real time. At hover, the Reynolds number (normalized by tip speed) for the presented test case was calculated to be between 13,000 and 14,000. 

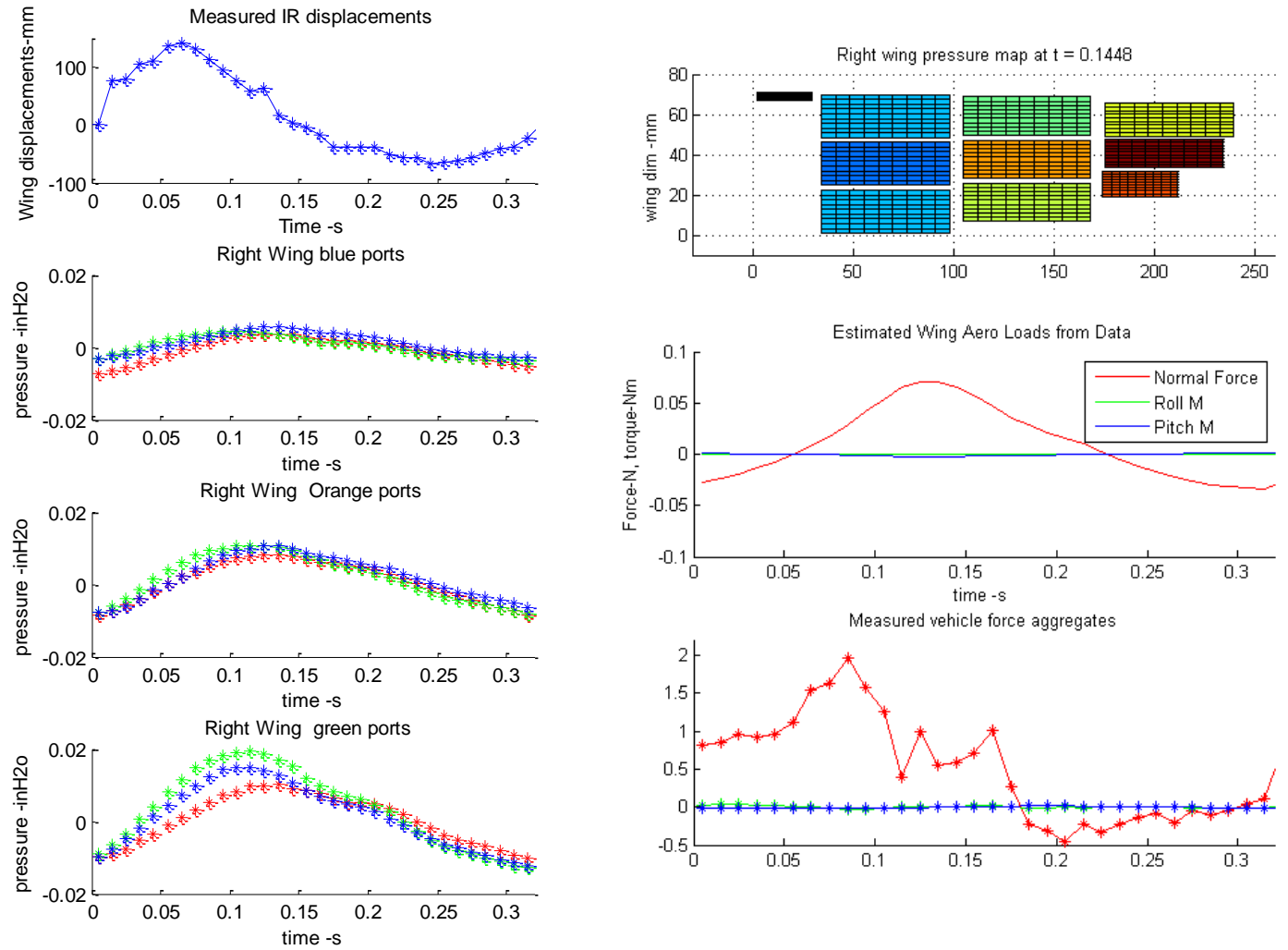

Figure 13: Elliptical Wings, 3Hz with no free-stream: Pressure and Force histories

Other than the much smaller pressure differential magnitudes at a standstill, it can be noted that there is no indication of a suction peak close to the quarter chord point as the flow across the leading edge is separated. At the outer sections, it can be observed that the pressure measurements near the center of the wing gave higher values than the measurements at either edge. The computed forces also appear to comprise a small portion of the loads being generated.

The following test results were acquired with a $3.2 \mathrm{~ms}$ free stream velocity and a $3.3 \mathrm{~Hz}$ flapping frequency. With the measured $26 \mathrm{~mm}$ stroke amplitude of the elliptical wings, this gave a Strouhal number of 0.27. 

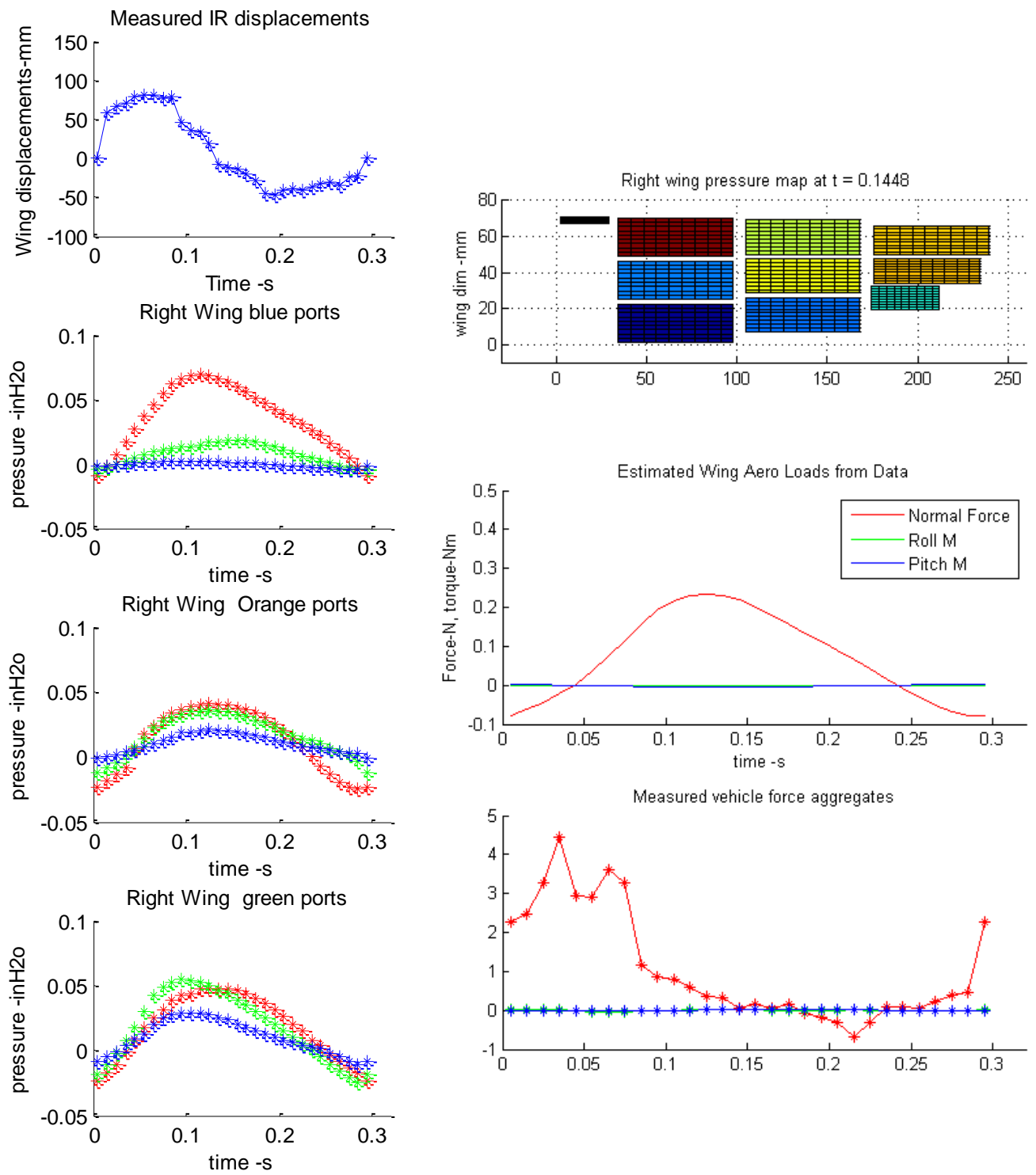

Figure 14: Elliptical Wings at $\mathrm{St}=0.27,3.3 \mathrm{~Hz}$ with $3.2 \mathrm{~ms}$ free-stream: Pressure and Force histories

At a $3.2 \mathrm{~ms}$ free stream, it was observed that the inboard 'blue' pressure histories appeared to regain the characteristics associated with attached flow as indicated by a large leading edge suction peak on downstrokes. Further outboard however, the pressure readings suggest that the flow is separated with the highest pressure being recorded near the center of the wing instead of near the leading edge. It can be noted that the difference in recorded forces at hover and $3 \mathrm{~m} / \mathrm{s}$ cannot be fully accounted for with the pressure readings alone, suggesting a systematic error associated with mounting or fouling of the pressure lines. These potential problems will be further investigated once inertial forces can be isolated.

\section{Conclusions and Future Work}

This paper has investigated the possibility of implementing a distributed pressure sensor network embedded in flapping wings to experimentally characterize flapping applied aerodynamic loads in real-time. Such sensing capability will facilitate flapping wing model validation and if miniaturized provide real-time aerodynamic feedback 
to an ornithopter autopilot. In this work, we constructed two pairs of instrumented flapping wings, one with embedded pressure ports $n$ air data system capable of of high speed pressure measurements taken over the surface of a flapping wing was developed and tested. A series of exploratory wind tunnel tests were run, the data was processed and analyzed. The sensing techniques were found to offer reliable measurements of the pressure fields. Further development on characterizing the flap stroke and making quantitative comparisons to numerical solutions are required.

Future work is required before a forward flight controller based on pressure feedback can be realized. The foremost hardware limitation we identified was the limited and mechanically-loose flap stroke of the existing Cybird mechanisms. Given wear over time, it was challenging to properly characterize the flapping angle of the root over our test series, and discrepancies in tracking the flap stroke can lead to inaccuracies in extrapolated models of aerodynamic forces as a function of stroke geometry. New mechanics that will be capable of wing pitch as well as flapping actuation are currently being developed and will be used in future testing. Due to the need to embed pressure tubing, thickness of the flat airfoil pair is currently $7 \%$ of the root chord. New manufacturing techniques that involve pre-fabricating the pressure lines for minimal thickness are expected to reduce this value to under $5 \%$. In near-term future work, we also plan to acquire inertial load measurements in a vacuum chamber and will upgrade our IR distance sensors to improve stroke displacement measurements. Quantitative comparisons with unsteady vortex method code output are also planned.

\section{References}

1 J. Han, J. Lee, and D. Kim, “Bio-inspired Flapping UAV Design: A University Perspective," SPIE, Vol. 7295, 2009.

2 R. Harmon directed by J. Hubbard Jr., Aerodynamic Modelling of A Flapping Membrane Wing Using Motion Tracking Experiments, University of Maryland -2007 College Park.

3 D. Yeo, J. Henderson, and E Atkins, "An Aerodynamic Data System for Small Hovering Fixed-Wing UAS," Proc. AIAA Guidance, Navigation, and Control Conference, 10-13 August 2009. (AIAA 2009-5756)

4 U. Gulcat, "Propulsive Force of a Flexible Flapping Thin Airfoil," Journal of Aircraft, Vol.46, No.2, MarchApril 2009.

5 M. A. Ashraf1, J. Young, and J. C. S. Lai. "Effect of Airfoil Thickness, Camber and Reynolds Number on Plunging Airfoil Propulsion," Proc. 47th AIAA Aerospace Sciences Meeting, January 2009. (AIAA 2009-1274)

6 A. Paranjape and S.-J. Chung, "Flight Mechanics of a Tail-less Articulated Wing Aircraft," Proc. AIAA Atmospheric Flight Mechanics Conference, Toronto, Canada, Aug. 2010. (AIAA-2010-7633)

7 A. Chakravarthy, A. Paranjape, and S.-J. Chung, "Control Law Design for Perching an Agile MAV with Articulated Wings," Proc. AIAA Atmospheric Flight Mechanics Conference, Toronto, Canada, Aug. 2010. (AIAA-2010-7934)

8 B. Nickless, Dynamic Modeling and Position Control of a Piezoelectric Flextensional Actuator, MS Thesis, University of Maryland - College Park, November 2008.

9 D. Kim, H.Kim, J.Han, and K, Kwon. "Experimental Investigation on the Aerodynamic Characteristics of a Biomimetic Flapping Wing with Macro-Fiber-Composites". Journal of Intelligent Material Systems and Structures, vol. 19, no. 3, 2008.

10 J. Grauer and J. Hubbard Jr. "Development of a Sensor Suite for a Flapping-Wing UAV Platform," Proc. $46^{\text {th }}$ AIAA Aerospace Sciences Meeting, January 2008. (AIAA 2008-224)

11 Z. Jackowski directed by R. Tedrake, Design and Construction of an Autonomous Ornithopter, MS Thesis, Massachusetts Institute of Technology, June 2009.

12 H.Takahashi, Y.Aoyama, K.Ohsawa, H. Tanaka, E. Iwase, K. Matsumoto, I.Shimoyama, "Differential pressure measurement using a free flying insect-like ornithopter with a MEMs sensor" Bioinspiration and Biomimetics, vol. 5 no. 3, 036005, 2010.

13 J. Katz, Low Speed Aerodynamics. Cambridge University Press, ISBN-10: 05216655232001

14 S.Ansari, R.Zbibowski, and K.Knowles, "Non-linear Unsteady Aerodynamic Model for Insect-like Flapping wings in the Hover. Part 1" Proceedings of the Institution of Mechanical Engineers, Part G: Journal of Aerospace Engineering, vol. 220, no. 2, pp. 61-83, 2006.

15 J. Katz. "A discrete vortex method for the non-steady separated flow over an airfoil." Journal of Fluid Mechanics, vol. 102, pp. 315-328, 1981.

16 D.J.Willis, J.Peraire and J.K.White, "FastAero - a Precorrected FFT - Fast Multipole Tree Steady and Unsteady Potential Flow Solver," presented at the SMA Symposium, Singapore 2005. 
17 D. J. Willis, P.-O.Persson, M.Drela, K.S.Breuer, S.M.Swartz, and J. Peraire, "A Computational Framework for Fluid Structure Interaction in Biologically-Inspired Flapping Flight," in Proceedings of the 25th AIAA Applied Aerodynamics Conference, Miami, FL, 2007.

18 W.Shyy, Y. Lian, J. Tang, D. Viieru, and H. Liu, Aerodynamics of Low Reynolds Number Flyers, Cambridge University Press, 2007. 